\title{
Receptor determinant analogues for all 9-O-Ac-sialoglycan-recognizing Corona viruses
}

\author{
Pratibha Preeti Maurya \\ Computational Biology for Biochemical Experiments (CBBE), Lucknow-21, India \\ Email: ppmcbbe@gmail.com
}

\begin{abstract}
In present study, analogues of receptor determinants were identified, which can mimic 9-O-Acsialoglycan-recognition by viruses and its usage in antiviral. It is well known that receptor determinants are part of host cell surface receptors which are recognized by virus surface glycoprotein as the first interaction of the target cell. This recognition governs the further processes of viral infection. Similar to other influenza viruses, Corona virus also processes through mechanisms of host interaction. This hostvirus interaction involves a conserved domain at interaction component of virus, which is known to be a key component during the process of virus infection. Therefore in the present study profile of possible Pharmacophore of conserved domain was used as a filter to identify analogues of receptor determinant from database ZINC database. The identified molecules were re-observed among the applicability domain defined by existing antiviral drugs as well as natural existing receptor determinant with sialic acid. The identified molecule needs to be further evaluated through in-vitro experiments.
\end{abstract}

Keywords: Corona; Determinant; Pharmacophore; Spike; Virus

\section{INTRODUCTION}

Viruses are responsible for various human diseases. Influenza is one of the conditions caused by viruses. Corona viruses also cause influenza. For influenza antiviral activity, different mechanisms have been developed such as: inhibition of ACE, inhibition of RNA expression, and barricading between virus and host cell etc. Out of these mechanisms, inhibition of interaction between host and virus is one of the concerned mechanisms. Such mechanisms of interaction involve the host cell surface Receptor which is recognized by the glycoprotein at the surface of the virus. The molecular component which is recognized by glycoproteins is called Receptor Determinants. Sialic Acid is at the base of Receptor Determinants within a groove of spike glycoprotein of Corona viruses (Schwegmann-Weßels and Herrler, 2006). Conservation of residual arrangement is found in the receptor-binding groove which recognizes 9-O-Acsialoglycan from the host cell surface (Alejandra Tortorici et al., 2019). This conservation can be used for 
identification of receptor-determinant-analogues through filtering of existing molecules on the basis of pharmacophore features derived from conserved domain. These analogues can act as a Receptor Determinant for binding but not for infection by virus (Brossmer et al., 1993). 3D structure description can be accessed from PDB 6NZK.

In general, pharmacophore mapping is the concept of generation of a profile based on a set of small molecules against a specific binding site. In this process a set 3D structure of small molecules are considered. Each molecule contains a specific 3D orientation of combination of pharmacophore features as hydrogen bond acceptor, hydrogen bond Donor, Negative Ion, aromatic ring hydrophobic areas etc. Data for these features are collected from each of the molecules of the set and is further summarized into an average combination of these 3D features called a pharmacophore map. This pharmacophore map can be used to filter those molecules from a library of compounds which will contain similar pharmacophore features for binding with that specific receptor. Beyond the generation of pharmacophores from a set of small molecules, multiple attempts had been made to define the pharmacophore directly on the basis of binding site at target protein. Such pharmacophores are called receptor defined pharmacophores. These are defined on the basis of 3D orientation of residues available in a binding pocket. Such binding pocket or binding sites should be the conserved areas within the hydrophobic locations of 3D structure of proteins. These binding pockets can be directly accessed from the PDB structure of the protein. The receptor defined filter can be directly used for screening of large databases to identify most suitable small molecules. Considering the above aspects, in present study, Receptor determinant analogues have been identified for all 9-O-Ac-sialoglycan recognizing Corona viruses.

\section{MATERIALS \& METHOD}

To identify the possible analogues for receptor determinant for interaction of corona virus with host, three basic sub-processes were involved: (i) pharmacophore for receptor determinant was defined; (ii) ZINC database was screened through pharmacophore; and (iii) screened out molecules were further visualized on the ground of applicability domain defined by known antiviral drugs.

Pharmacophore, screening \& evaluation: PDB: 6NZK was used to identify conserved groove details of receptor determinant binding site at the surface of virus. Groove's conserved components were used to define pharmacophore, which was further implemented as a filter for screening of ZINC Database. Defining Pharmacophore for receptor-binding groove, based on 'Pharmacophore Screening of the Protein Data Bank for Specific Binding Site Chemistry' (Campagna-Slater et al., 2010), using PharmaGist 
(Schneidman-Duhovny et al., 2008). Other methods are also known for pharmacophore mapping of protein domain. Library screening: Pharmacophore information was used as input for ZINCpharmer (Koes and Camacho, 2012) as filter to screen molecules in ZINC database. Furthermore, identified molecules were visualized within the applicability domain defined on the basis of existing known antiviral molecules. Applicability domain was plotted with two basic physicochemical properties LogP \& Molecular weight.

\section{RESULTS \& DISCUSSION}

Sialic acid based receptor determinants are the core for defining analogues because the influenza viruses are characterized by one of the surface glycoprotein which has capability to recognize the receptor with $\mathrm{N}$-acetyl-9-O-acetylneuramic acid. The receptor destroying enzyme acetylesterase releases the 9-Oacetyl group of Sialic acid. Here the attention is that if analogues are designed to mimic this receptor determinant then acetylesterase will not be able to act upon the analogues, which will father stop the signaling process for entry of virus into the host. The point to be noted is that the analogues will act as receptor determinant, but these analogues will not defend antiviral performers. In general the Sialic acids are also known as receptor determinants for Corona viruses.

Glycoprotein's groove conserved domain was extracted from PDB 6NZK. Domain contains residues with Asn27, Asp28, Lys29, Asp30, Thr31, Leu80, Lys81, Ser83, Va184, Leu85, Leu86, Trp90, Phe95. This has been divided into two sub-domains P1 \& P2 (Figure 1). P1 contains a hydrophobic pocket with residues at serial no. $85,86, \&$ 90; while P2 sub-domain contains residues with serial no. 80, 90, \& 95. Wild receptor determinant 9-O-Ac-sialoglycan stacks into the groove. The pockets with conserved residues were used to define the pharmacophore features. The pharmacophore features included four basic components namely hydrogen bond acceptor, hydrogen bond Donor, hydrophobic areas and aromatic rings (Figure 2). 3D orientations of these four features were found to be concerned for defining a pharmacophore equivalent to multiple analogues of receptor determinant. It was observed that the distance among pharmacophore features were ranging between 1.68 to 3.9 angstrom; and hydrophobic locations were found to be closest, while hydrogen bond acceptors and aromatic rings were found to be distantly located. This pharmacophore was found to be very selective, since it filtered out only 10 possible structures from the zinc database. These identified molecules were marked as C1-10 (Figure 3).

To visualize the possibilities of existence of identified molecules within the applicability domain of antiviral drugs; the screened out molecules and antiviral drugs were plotted on the ground of $\operatorname{LogP} \&$ Molecular Weight. It was observed that the identified molecules were also lying within the same applicability domain defined for antiviral activity. The majority of them belong to the negative values of 
the $\log \mathrm{P}$ area. $\log \mathrm{P}$ is one of the pharmacokinetics properties which is used to define the behavior of drugs during the passive diffusion are carrier mediated uptake. Therefore this property is used to establish an applicability domain for or a set of molecules concerned for a specific purpose. The queried molecule can be accessed on the basis of boundaries of applicability domain defined by LogP and other features defining pharmacokinetics. $\log \mathrm{P}$ is also well known for evaluation of Lipophilicity of chemical compounds, and it is also a part of Lipinski's rule of 5. This property is directly linked with $\mathrm{pH}$ dependent behavior of compounds within the environment of the system therefore it becomes an important parameter during the evaluation of the drug development process. Another aspect which is considered for defining the generalized applicability domain is molecular weight of the compound. Therefore LogP and Molecular weight provide a ground for establishment of a frame of evaluation for any small molecule queried for their existence in the previously known standard drugs (Figure 4).
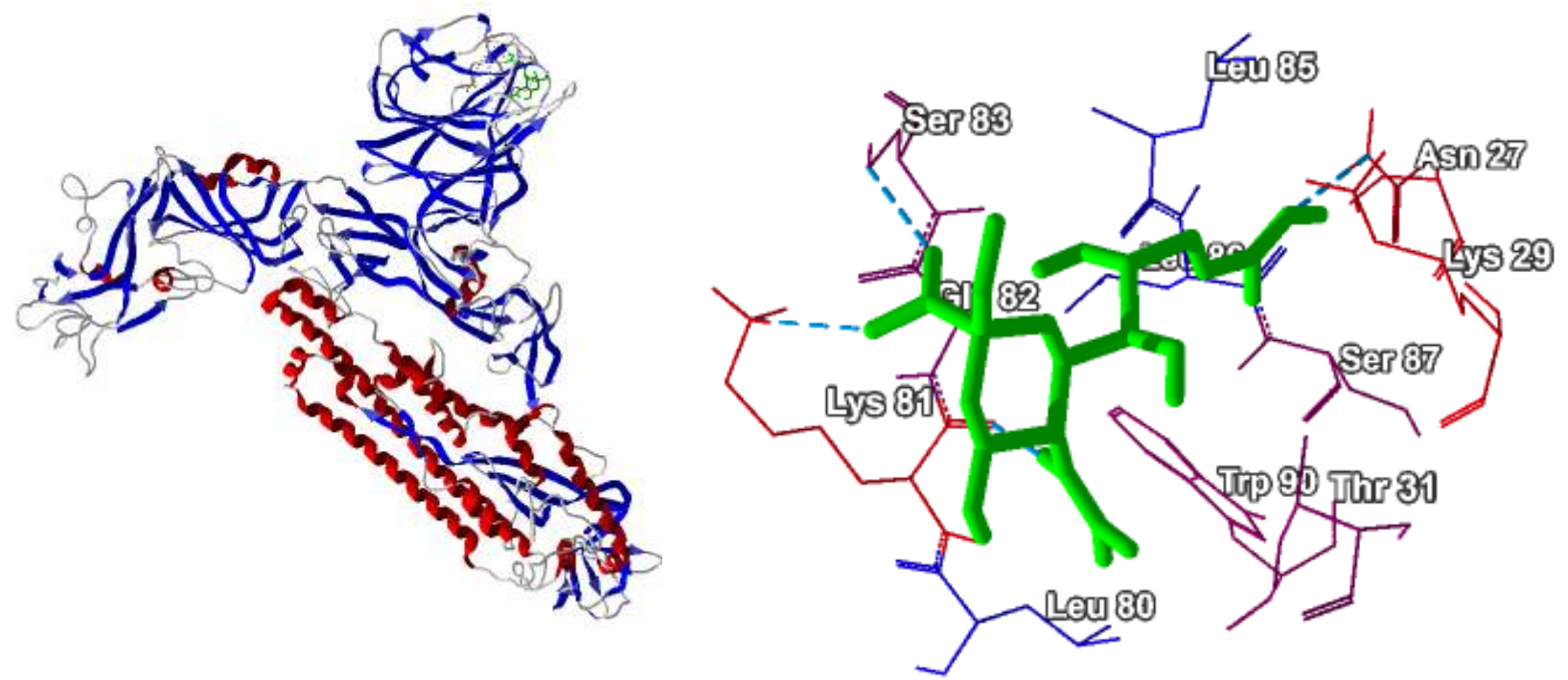

Figure 1. Conserved receptor-binding groove from PDB 6NZK. It is shown that 9-O-Ac-sialoglycan being integrated into a conserved groove of virus-spike.

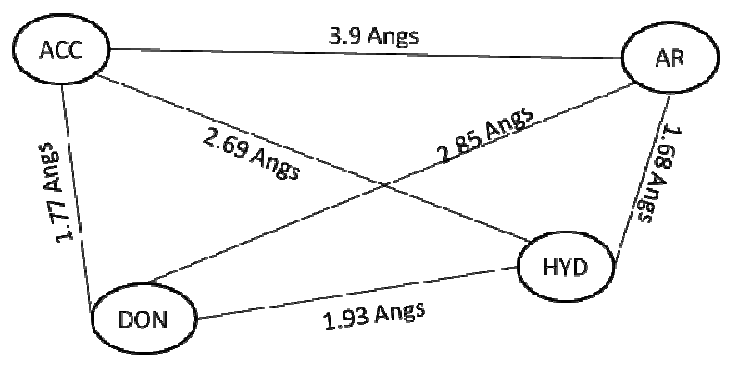

\begin{tabular}{|c|c|c|c|c|}
\cline { 2 - 5 } \multicolumn{1}{c|}{} & ACC & AR & DON & HYD \\
\hline ACC & 0 & 3.977329 & 1.77213 & 2.693543 \\
\hline AR & & 0 & 2.858369 & 1.687619 \\
\hline DON & & & 0 & 1.932673 \\
\hline HYD & & & & 0 \\
\hline
\end{tabular}


Figure 2. Pharmacophore distance $(\AA)$ map. Pharmacophore was defined on the basis of conserved domain.<smiles>CO[C@]1(C(=O)O)C[C@@H](O)[C@H](NC(C)=O)[C@H]([C@H](O)[C@H](O)COC(C)O)O1</smiles>

5-Acetylamino-6-[1,2-dihydroxy-3(1-hydroxy-ethoxy)-propyl]-4hydroxy-2-methoxy-tetrahydropyran-2-carboxylic acid<smiles>NC(=O)NC(=O)N1CC=CN1</smiles>

(C2)<smiles></smiles>

(C5)<smiles>CC(C)(C)C1=C[C@@H]2NC(N)N[C@@H](N)N2N1</smiles>

(C8)<smiles></smiles>

5-Acetylamino-2,2,4,7,8,9hexahydroxy-nonanoic acid (Sialic Acid)<smiles>N[C@@H]1NC(=O)NC2=C1NNN2</smiles>

(C3)<smiles>O=C1NC(=O)C2NCN(C(=O)O)C2N1</smiles>

(C6)<smiles>O=c1[nH]c2c(c(=O)[nH]1)OC(c1ccccc1)N2</smiles>

(C9)<smiles>C[C@@H]1NC2=C(NON2)NC1=O</smiles>

(C1) 


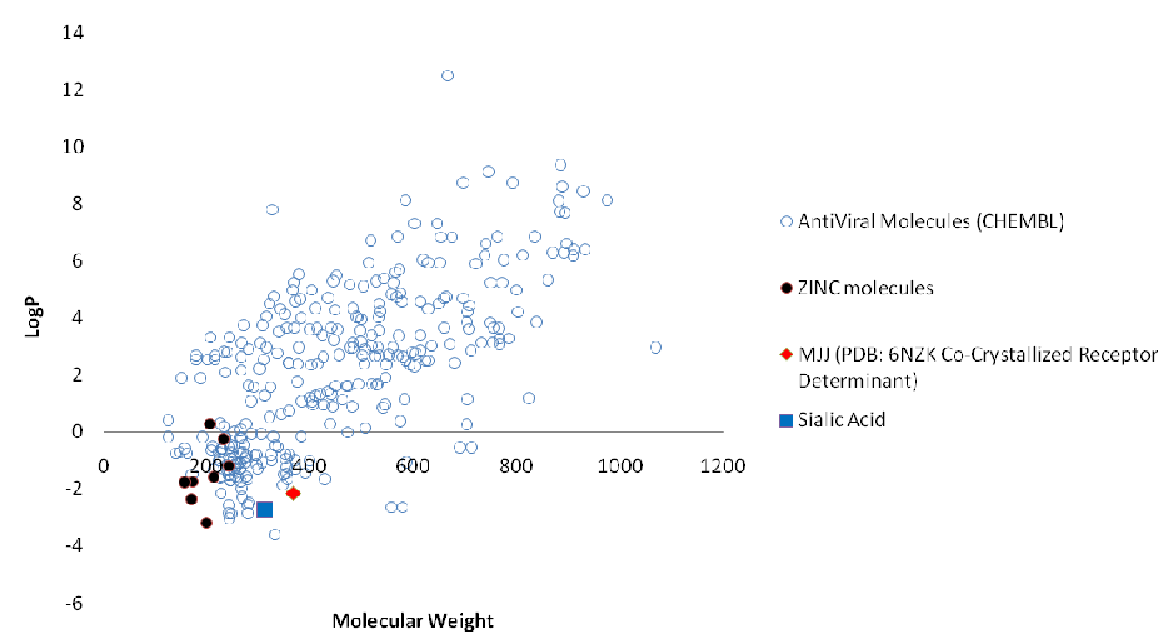

Figure 4. Screened molecules were co-plotted ( $\log \mathrm{P}$ vs. Molecular weight) with known antiviral drugs. Existence of analogues in the domain of antiviral compounds

\section{CONCLUSION}

Study was performed for identification of receptor determinant analogues for all 9-O-Ac-sialoglycanrecognizing Corona viruses. Pharmacophore based screening of ZINC Database was performed. As result 10 molecules were identified, they need to be further evaluated for prioritization \& antiviral performance.

\section{REFERENCES}

Alejandra Tortorici, M., Walls, A.C., Lang, Y., Wang, C., Li, Z., Koerhuis, D., Boons, G.J., Bosch, B.J., Rey, F.A., de Groot, R.J., Veesler, D., 2019. Structural basis for human coronavirus attachment to sialic acid receptors. Nat. Struct. Mol. Biol. 26, 481-489. https://doi.org/10.1038/s41594-019-0233y

Brossmer, R., Isecke, R., Herrler, G., 1993. A sialic acid analogue acting as a receptor determinant for binding but not for infection by influenza $C$ virus. FEBS Lett. 323. https://doi.org/10.1016/00145793(93)81456-A

Campagna-Slater, V., Arrowsmith, A.G., Zhao, Y., Schapira, M., 2010. Pharmacophore screening of the protein data bank for specific binding site chemistry. J. Chem. Inf. Model. 50. https://doi.org/10.1021/ci900427b

Koes, D.R., Camacho, C.J., 2012. ZINCPharmer: Pharmacophore search of the ZINC database. Nucleic Acids Res. 40. https://doi.org/10.1093/nar/gks378

Schneidman-Duhovny, D., Dror, O., Inbar, Y., Nussinov, R., Wolfson, H.J., 2008. PharmaGist: a webserver for ligand-based pharmacophore detection. Nucleic Acids Res. 36. https://doi.org/10.1093/nar/gkn187 
Schwegmann-Weßels, C., Herrler, G., 2006. Sialic acids as receptor determinants for coronaviruses. Glycoconj. J. https://doi.org/10.1007/s10719-006-5437-9 